Supplemental Information

\title{
Atmospheric deposition and annual flux of legacy perfluoroalkyl substances and replacement perfluoroalkyl ether carboxylic acids in Wilmington NC, USA
}

\author{
Megumi S. Shimizu1*, Rachael Mott ${ }^{1}$, Ariel Potter ${ }^{1}$, Jiaqi Zhou ${ }^{2}$, Karsten Baumann², Jason D. \\ Surratt $^{2,3}$, Barbara Turpin², G. Brooks Avery ${ }^{1}$, Jennifer Harfmann¹, Robert J. Kieber ${ }^{1}$, Ralph N. \\ Mead $^{1 *}$, Stephen A. Skrabal ${ }^{1}$, Joan D. Willey \\ 1. Department of Chemistry and Biochemistry, University of North Carolina, Wilmington, NC, \\ USA \\ 2. Department of Environmental Sciences and Engineering, Gillings School of Global Public \\ Health, University of North Carolina, Chapel Hill, NC, USA \\ 3. Department of Chemistry, University of North Carolina, Chapel Hill, NC, USA
}

\section{Sample Collection}

Wet deposition samples were collected on an event basis and stored in HDPE bottle(s) at $4{ }^{\circ} \mathrm{C}$ until extraction. The rain amount of each event was measured using a rain gauge at the sampling site. Dry deposition samples were collected usually bi-weekly. The N-CON 00-120 automatic deposition sampler consists of a side-by-side sample container, one for wet and the other for dry deposition, a motor-driven lid, and a motion sensor. When it is not raining, the dry deposition collector is uncovered and collecting a sample. When the motion sensor senses the rain, the lid moves from wet to dry side collecting the wet deposition. When rain stops, the lid moves back to the wet deposition side re-exposing the dry deposition collector. The dry deposition side stayed dry over the course of the sampling period, and the buckets were replaced at the end of the sampling. The sampling period for dry deposition was set to be 7 days initially and changed to 14 days, and the sampling periods range due to University closures and extreme storms (Table S1). When an extreme storm was expected, dry deposition buckets were retrieved regardless of sampling period to avoid contamination of rainwater. Note that this sampling method does not include dry deposition of the gas-phase components to soil or aqueous surfaces.

\section{Analytical standards}

The legacy and emerging PFASs studied in this research are listed in Table S2. Standards for native PFOA and PFOS were purchased from Synquest (Alachua, Florida). PFMOAA standard was purchased from Zerenex (Greater Manchester, UK). HFPO-DA was purchased from Apollo Scientific (Stockport, UK). PFMOBA standard was purchased from Aurum Pharmatech (Franklin Park, NJ), and HFPO-DA standard was purchased from Alfa Aesar (Haverhill, MA). The masslabeled internal standards, M-PFBA (perfluoro[ $\left.{ }^{13} \mathrm{C}_{4}\right]$ butanoic acid), M-PFHxA (perfluoro $\left[{ }^{13} \mathrm{C}_{6}\right]$ hexanoic acid), M-HFPO-DA (perfluoro $\left[{ }^{13} \mathrm{C}_{6}\right]$ propoxypropanoic acid), M-PFOA (perfluoro $\left[{ }^{13} \mathrm{C}_{8}\right]$ octanoic acid), and M-PFOS (perfluoro $\left[{ }^{13} \mathrm{C}_{8}\right]$ octane sulfonic acid) were all purchased from Wellington laboratories (Guelph, Canada). All the standards were individually diluted first in LCMS grade methanol and ammonium hydroxide $\left(\mathrm{NH}_{4} \mathrm{OH}\right)$ solution $(96 \%$ methanol, 4\% $\mathrm{NH}_{4} \mathrm{OH}$ ) and stored in clean 30-mL HDPE bottles. The standard mix and internal standard mix were prepared gravimetrically in a 1:1 2mM ammonium acetate $\left(\mathrm{NH}_{4} \mathrm{OAc}\right)$ methanol and water solution. 


\section{PFAS quantification}

PFAS separation and quantification was performed on a Sciex Exion LC equipped with a Luna Omega PC C18 $(2.1 \times 50 \mathrm{~mm}, 1.6 \mu \mathrm{m}$, Phenomenex $)$ and AB Sciex Qtrap 4000 triple quadrupole mass spectrometer. The mobile phase gradient is shown in Table S3. Electrospray ionization (ESI) in negative mode was used, and the ion spray voltage was kept at $-4200 \mathrm{~V}$. The ion source temperature was set to $500^{\circ} \mathrm{C}$, and the source gas flow was maintained at 60 psi. PFMOPrA and PMPA share parent ion and fragment ion masses. Because the separation of PFMOPrA and PMPA was not achieved under the LC conditions used in this method, the values reported are the sum of two compounds. Calibration standards and quality control samples were spiked with isotopically labeled internal standards. Quantification was performed using isotope dilution. Analytes lacking an analogous labeled standard were quantified using the internal standard with the closest retention time (Table S4). The precursor-product ion multiple reaction monitoring (MRM) transitions are shown in Table S4. Calibration curves dissolved in $2 \mathrm{mM} \mathrm{NH} 4 \mathrm{OAc}$ methanol/water (25/75), consisting of minimal four standards, were linear with $r^{2}$ values greater than 0.99. Besides the field blank, method blanks and instrument blanks were included during the analyses. Following Sun et al., ${ }^{1}$ and Pan et al., ${ }^{2}$ the limit of quantification (LOQ) was defined as the lowest point of the standard curve where the calculated concentration was 70 to $130 \%$ of expected using the linear equation. From the LOQ concentration for each of the targeted compounds, the mass on column was calculated based upon injection volume and used as the limit of quantification for all samples collected from wet and dry deposition. All targeted compounds mass on column in both wet and dry deposition were compared back to this value and was considered quantifiable if above it. Using typical volume of wet deposition extracted and dry deposition collection periods, the LOQ expressed as a concentration (ng/L) and flux (ng/ $\mathrm{m}^{2} /$ day) are shown for each targeted compound in Table S6. However, the volume of wet deposition collected, and dry deposition deployment periods varied resulted in non-uniform sample sizes, therefore, mass on column was used as the limit of quantification metric. The volume of wet deposition extracted, and dry deposition collection periods are shown in Tables S1 and S2.

\section{Quality Assurance and Control (QA/QC)}

Field blanks for wet deposition were taken by filling 1-L HDPE bottles with Milli-Q water and stored under the same conditions as the wet deposition samples. Field blanks for dry deposition were conducted by leaving the sampling buckets with the lids on at the sampling site, and then the buckets were taken back to the lab at the time of sample collection. Long-term degradation studies on the targeted PFASs over 11 weeks at room temperature in HDPE buckets demonstrated no significant difference in concentrations of targeted analytes ( $p$-values $>0.2 ; t$ test). Method blanks consisting of Milli-Q water were processed in tandem with every batch of five samples. Method blanks were quantified along with the unknown samples by LC-MS/MS and if any targeted compounds were detected above the LOQ in the blank, it was subtracted from that corresponding batch of samples. Out of thirty-nine wet deposition samples extracted, blank subtraction occurred $28 \%, 46 \%, 33 \%, 56 \%, 2 \%$ and $48 \%$ of the time for PFMOAA, PFMOBA, PFMOPrA/PMPA, HFPO-DA, PFOA and PFOS respectively. Twenty five dry deposition samples were collected for this study and only PFOS, HFPO-DA and PFMOBA required blank subtraction at $32 \%, 12 \%$ and $4 \%$ respectively. The remaining targeted compounds were not above the LOQ in any of the corresponding batch method blanks. 
Spike Recoveries were calculated based on a matrix-matched spike extraction. A matrixmatched sample $(3000 \mathrm{~mL}$ of wet deposition and 3 buckets of dry deposition with $700 \mathrm{~mL}$ of MilliQ water added to each) were aliquoted into three. Two of the aliquots were extracted as described above without the addition of standards and (matrix blank and matrix spike A), and one of the aliquots was spiked with known amounts of the standards (matrix spike B) before extraction. After the elution of the PFASs from the SPE cartridges, the internal standards were added to the eluate of all three and the same known amount of the standards were added to the matrix spike A. The area ratio of the matrix spike $\mathrm{B}$ was divided by that of the matrix spike $\mathrm{A}$ to give a recovery determination. The equation used for the calculation is shown below. The average spike recoveries of PFAS in wet and dry deposition ranged from 68\% to 102\% (Table S5). Spike levels of $30 \mathrm{ng}$ and $0.9 \mathrm{ng}$ were tested.

Where

$$
\text { Recovery }(\%)=(\mathrm{SB}-\mathrm{BLK}) /(\mathrm{SA}-\mathrm{BLK}) \times 100
$$

$\mathrm{SB}=$ response for SPE extract of matrix spiked before SPE

$\mathrm{SA}=$ response for SPE extract of matrix spiked after SPE

$\mathrm{BLK}=$ response for SPE extract of matrix without spike

\section{HYSPLIT Airmass back trajectory models}

Air-mass back trajectories were generated for each rainwater sample collected in Wilmington, NC, using the Hybrid Single Particle Lagrangian Integrated Trajectory (HYSPLIT) model. ${ }^{3}$ The back trajectories were calculated starting at the end of the precipitation event for a 120-h hind-cast at an altitude of 100, 500, and $1000 \mathrm{~m}$. Rain events were categorized into five air mass back trajectories, including terrestrial, mixed, marine east, marine south, and coastal. ${ }^{4}$ Terrestrial air masses travel across the land; mixed air masses travel over land and water; marine air masses travel over the ocean for at least 48 hours.

\section{Supporting Analyses}

A Ross electrode with low ionic strength buffers was used for $\mathrm{pH}$ analysis. Organic carbon content in rainwater samples was determined with a Shimadzu TOC 5000 carbon analyzer (Shimadzu, Kyoto, Japan) equipped with an ASI 5000 auto- sampler ${ }^{5}$. Inorganic anions ( $\mathrm{Cl}^{-}$, $\mathrm{NO}_{3}{ }^{-}$, and $\mathrm{SO}_{4}{ }^{2-}$ ) were analyzed using suppressed ion chromatography with Dionex ICS-1600 (Thermo Scientific).

\section{Statistical Analysis}

Correlation tests among PFAS concentration/flux in wet deposition and other chemical measurements were performed using R statistical software (R version 3.6.3). Statistical significance was set at $\mathrm{p}<0.05$. Due to the presence of data below the limit of quantification, functions from the "NADA" package was used to perform a non-parametric correlation test, Kendall's tau b. ${ }^{6}$ 
Table S1. Wet deposition sampling dates, amount and volume of wet deposition extracted for PFAS analysis. All of the wet deposition collected was extracted for analysis for each event.

\begin{tabular}{|c|c|c|c|}
\hline $\begin{array}{c}\text { Wet Deposition } \\
\text { sampleID }\end{array}$ & Date & $\begin{array}{l}\text { Wet Deposition } \\
\text { amount (mm) }\end{array}$ & Volume Extracted (L) \\
\hline E1876 & $2018-12-08$ & 24.6 & 0.98 \\
\hline E1879 & $2018-12-20$ & 31.8 & 1.0 \\
\hline E1881 & $2018-12-28$ & 22.4 & 0.97 \\
\hline E1882 & $2018-12-29$ & 3.6 & 0.54 \\
\hline E1884 & 2019-01-04 & 13.7 & 1.0 \\
\hline E1887 & 2019-01-20 & 4.3 & 1.0 \\
\hline E1889 & 2019-01-28 & 0.3 & 0.02 \\
\hline E1897 & 2019-03-01 & 8.6 & 1.0 \\
\hline E1898 & 2019-03-03 & 34.8 & 1.0 \\
\hline E1900 & 2019-03-08 & 4.32 & 0.24 \\
\hline E1902 & 2019-03-26 & 0.5 & 0.07 \\
\hline E1904 & 2019-04-05 & 28.7 & 1.0 \\
\hline E1905 & 2019-04-09 & 2.03 & 0.12 \\
\hline E1909 & 2019-05-05 & 2.8 & 0.16 \\
\hline E1912 & 2019-06-02 & 29.2 & 2.0 \\
\hline E1914 & 2019-06-06 & 1.5 & 0.5 \\
\hline E1915 & 2019-06-07 & 7.87 & 0.76 \\
\hline E1916 & 2019-06-09 & 13.21 & 0.84 \\
\hline E1917 & 2019-06-12 & 3.3 & 0.5 \\
\hline E1921 & 2019-07-03 & 54.1 & 1.0 \\
\hline E1923 & 2019-07-05 & 6.6 & 0.9 \\
\hline E1924 & 2019-07-09 & 25.4 & 1.0 \\
\hline E1925 & 2019-07-11 & 25.2 & 1.0 \\
\hline E1926 & 2019-07-23 & 1.5 & 0.19 \\
\hline E1927 & 2019-08-02 & 6.9 & 1.0 \\
\hline E1929 & 2019-08-10 & 11.7 & 1.0 \\
\hline E1933 & 2019-09-01 & 20.3 & 1.0 \\
\hline E1934 & 2019-09-05 & 217.93 & 1.0 \\
\hline E1935 & 2019-09-09 & 13.46 & 1.0 \\
\hline E1936 & 2019-09-27 & 6.1 & 1.0 \\
\hline E1938 & $2019-10-13$ & 28.45 & 1.0 \\
\hline E1939 & 2019-10-19 & 42.16 & 1.0 \\
\hline E1943 & 2019-11-08 & 27.94 & 1.0 \\
\hline E1945 & 2019-11-15 & 44.45 & 1.0 \\
\hline E1947 & $2019-11-23$ & 5.33 & 0.5 \\
\hline E1948 & 2019-12-01 & 4.32 & 0.8 \\
\hline E1949 & $2019-12-08$ & 21.59 & 1.0 \\
\hline E1950 & $2019-12-13$ & 20.57 & 1.0 \\
\hline E1952 & 2019-12-30 & 8.64 & 1.0 \\
\hline
\end{tabular}


Table S2. Dry deposition sampling dates and sampling period for each event. All of the dry deposition collected was extracted for PFAS analysis for each event.

\begin{tabular}{ccc}
\hline $\begin{array}{c}\text { Dry Deposition } \\
\text { Sample ID }\end{array}$ & Sate & Sampling period (days) \\
\hline DDW3 & $2018-12-21$ & 8 \\
DDW5 & $2019-01-18$ & 14 \\
DDW6 & $2019-01-25$ & 7 \\
DDW7 & $2019-02-01$ & 7 \\
DDW8 & $2019-02-15$ & 14 \\
DDW9 & $2019-02-22$ & 7 \\
DDW10 & $2019-03-08$ & 14 \\
DDW11 & $2019-03-29$ & 21 \\
DDW12 & $2019-04-07$ & 9 \\
DDW13 & $2019-04-12$ & 5 \\
DDW14 & $2019-04-28$ & 16 \\
DDW15 & $2019-05-24$ & 26 \\
DDW16 & $2019-06-11$ & 18 \\
DDW17 & $2019-06-25$ & 14 \\
DDW18 & $2019-07-23$ & 28 \\
DDW19 & $2019-08-06$ & 14 \\
DDW20 & $2019-08-20$ & 14 \\
DDW21 & $2019-09-03$ & 14 \\
DDW23 & $2019-10-01$ & 14 \\
DDW24 & $2019-10-15$ & 14 \\
DDW25 & $2019-11-01$ & 17 \\
DDW26 & $2019-11-24$ & 23 \\
DDW27 & $2019-12-09$ & 17 \\
DDW28 & $2020-01-12$ & \\
DDW29 & $2020-01-29$ & \\
\hline
\end{tabular}


Table S3. Targeted PFAS analytes quantified in this research.

\begin{tabular}{llll}
\hline \multirow{2}{*}{ Compound Name } & \multirow{2}{*}{ Formula } & $\begin{array}{l}\text { Molecular } \\
\text { Weight }\end{array}$ & Cas. No. \\
\hline Perfluoro-2-methoxyacetic acid (PFMOAA) & $\mathrm{C}_{3} \mathrm{HF}_{5} \mathrm{O}_{3}$ & 180.0 & $674-13-5$ \\
Perfluoro-3-methoxypropanoic acid (PFMOPrA)/ & $\mathrm{C}_{4} \mathrm{HF}_{7} \mathrm{O}_{3}$ & 230.0 & $377-73-1 /$ \\
Perfluoro-2-methoxypropanoic acid (PMPA) & $\mathrm{C}_{5} \mathrm{HF}_{9} \mathrm{O}_{3}$ & 280.0 & $13140-29-9$ \\
Perfluoro-4-methoxybutanoic acid (PFMOBA) & $863090-89-5$ \\
Hexafluoropropylene Oxide Dimer Acid (HFPO-DA) & $\mathrm{C}_{6} \mathrm{HF}_{11} \mathrm{O}_{3}$ & 330.1 & $13252-13-6$ \\
Perfluorooctanoic acid (PFOA) & $\mathrm{C}_{8} \mathrm{HF}_{15} \mathrm{O}_{2}$ & 414.1 & $335-67-1$ \\
Perfluorooctane sulfonic acid (PFOS) & $\mathrm{C}_{8} \mathrm{HF}_{17} \mathrm{SO}_{3}$ & 500.1 & $1763-23-1$ \\
\hline
\end{tabular}

Table S4. The mobile phase gradient program for quantitative analysis.

\begin{tabular}{llll}
\hline $\begin{array}{l}\text { Time } \\
(\mathrm{min})\end{array}$ & $\begin{array}{l}\mathrm{A} \\
(\%)\end{array}$ & $\begin{array}{l}\mathrm{B} \\
(\%)\end{array}$ & $\begin{array}{l}\text { Flow rate } \\
(\mathrm{mL} / \mathrm{min})\end{array}$ \\
\hline 0 & 95 & 5 & 0.4 \\
0.1 & 45 & 55 & 0.4 \\
4.5 & 1 & 99 & 0.4 \\
8.0 & 1 & 99 & 0.4 \\
8.5 & 95 & 5 & 0.4 \\
10.0 & 95 & 5 & 0.4 \\
\hline
\end{tabular}

Table S5. MS transitions, MRM parameters (CE: collision energy, DP: declustering potential, CXP: cell exit potential, EP: entrance potential), and internal standards for PFAS analysis.

\begin{tabular}{cccccccc}
\hline Analyte & $\begin{array}{c}\text { Isolation } \\
\text { mass }\end{array}$ & $\begin{array}{c}\text { Quantifier } \\
\text { mass }\end{array}$ & $\begin{array}{c}\text { CE } \\
\text { (volt) }\end{array}$ & $\begin{array}{c}\text { DP } \\
\text { (volt) }\end{array}$ & $\begin{array}{c}\text { CXP } \\
\text { (volt) }\end{array}$ & $\begin{array}{c}\text { EP } \\
\text { (volt) }\end{array}$ & Internal Standard \\
\hline PFMOAA & 179 & 85 & -15 & -30 & -5 & -10 & M-PFBA \\
PFMOPrA/ & 229 & 85 & -20 & -50 & -5 & -10 & M-PFBA \\
PMPA & & & & & & -14 & M-PFHxA \\
PFMOBA & 279 & 85 & -25 & -40 & -5 & -14 & M-HFPO-DA \\
HFPO-DA & 329 & 285 & -15 & -30 & -5 & -10 & M-PFOA \\
PFOA & 413 & 369 & -14 & -50 & -6 & -10 & M-PFOS \\
PFOS & 499 & 99 & -70 & -120 & -14 & -14 & \\
M-PFBA & 217 & 172 & -15 & -40 & -7 & -14 & \\
M-PFHxA & 315 & 270 & -15 & -48 & -12 & -10 & \\
M-HFPO-DA & 332 & 169 & -18 & -30 & -1 & -10 & \\
M-PFOA & 421 & 376 & -14 & -50 & -6 & -10 & \\
M-PFOS & 507 & 99 & -70 & -120 & -14 & -14 & \\
\hline
\end{tabular}


Table S6. Compound-specific quantification limits (QLs) and spike recoveries in wet and dry deposition. Given the variable volume of wet deposition and the different dry deposition duration of deployment, mass on column QLs are presented and defined as the lowest point of the standard curve where the calculated concentration was 70 to $130 \%$ of expected using the linear equation.

\begin{tabular}{|c|c|c|c|c|c|c|}
\hline & PFMOAA & $\begin{array}{c}\text { PFMOPrA/ } \\
\text { PMPA }\end{array}$ & PFMOBA & HFPO-DA & PFOA & PFOS \\
\hline \multicolumn{7}{|c|}{ Quantification Limits } \\
\hline pg per injection & 3.8 & 4.3 & 4.0 & 4.0 & 3.6 & 4.2 \\
\hline $\begin{array}{l}\text { Wet deposition } \\
\left(n g L^{-1}\right)^{\mathrm{a}}\end{array}$ & 0.38 & 0.43 & 0.40 & 0.40 & 0.36 & 0.42 \\
\hline $\begin{array}{l}\text { Dry Deposition } \\
\left(\text { ng m }^{-2} \text { day }^{-1}\right)^{b}\end{array}$ & 0.14 & 0.15 & 0.16 & 0.15 & 0.13 & 0.16 \\
\hline \multicolumn{7}{|l|}{ Recoveries } \\
\hline $\begin{array}{l}\text { Wet Deposition } \\
(n=3)\end{array}$ & $94 \pm 18 \%$ & $94 \pm 7 \%$ & $85 \pm 5 \%$ & $80 \pm 5 \%$ & $100 \pm 11 \%$ & $87 \pm 9 \%$ \\
\hline $\begin{array}{l}\text { Dry Deposition } \\
(n=3)\end{array}$ & $94 \pm 10 \%$ & $101 \pm 11 \%$ & $98 \pm 17 \%$ & $98 \pm 32 \%$ & $102 \pm 13 \%$ & $68 \pm 39 \%$ \\
\hline
\end{tabular}

a One L of wet deposition extracted, $0.5 \mathrm{~mL}$ eluate, $20 \mu \mathrm{L}$ injection

b Three collection buckets pooled and deployed for 14 days, $0.5 \mathrm{~mL}$ eluate, $20 \mu \mathrm{L}$ injection 
Table S7. PFAS concentrations (ng $\mathrm{L}^{-1}$ ) in wet deposition and flux (ng $\mathrm{m}^{-2}$ day) in dry deposition.

\begin{tabular}{|c|c|c|c|c|c|c|c|c|c|c|}
\hline Location & location type & $\begin{array}{c}\text { Sampling } \\
\text { period }\end{array}$ & $n$ & PFMOAA & $\begin{array}{l}\text { PFMOPrA } \\
\text { /PMPA } \\
\end{array}$ & PFMOBA & $\begin{array}{l}\text { HFPO- } \\
\text { DA }\end{array}$ & PFOA & PFOS & References \\
\hline \multicolumn{11}{|l|}{ Wet Deposition } \\
\hline Wilmington, NC & urban/coastal & $\begin{array}{l}2018- \\
2019\end{array}$ & 24 & $\begin{array}{c}<\mathrm{MQL}- \\
32\end{array}$ & $\begin{array}{c}<\mathrm{MQL}- \\
14\end{array}$ & $\begin{array}{l}<\mathrm{MQL}- \\
16\end{array}$ & $\begin{array}{c}<\mathrm{MQL}- \\
28\end{array}$ & $\begin{array}{l}<\mathrm{MQL}- \\
\quad 4.6\end{array}$ & $\begin{array}{c}<\mathrm{MQL}- \\
100\end{array}$ & This Study \\
\hline $\begin{array}{l}\text { Ohilo, Indina, } \\
\text { Wyoming, USA }\end{array}$ & rural/suburban/urban & 2019 & 54 & n.a. & n.a. & n.a. & $\begin{array}{c}5 \times 10^{-4} \\
\quad-5\end{array}$ & $0.03-30$ & $0.07-50$ & 7 \\
\hline $\begin{array}{l}\text { Lakes Ontario, } \\
\text { Erie, Huron, } \\
\text { Superior, Canada }\end{array}$ & remote/rural & $\begin{array}{l}2006- \\
2018\end{array}$ & 447 & n.a. & n.a. & n.a. & n.a. & $\begin{array}{c}<\mathrm{MQL}- \\
11\end{array}$ & $\begin{array}{c}<\mathrm{MQL}- \\
14\end{array}$ & 8 \\
\hline Corvo, Portugal & remote/coastal & 2016 & 5 & n.a. & n.a. & n.a. & n.a. & $0.2-1.7$ & $0.6-1.3$ & 9 \\
\hline $\begin{array}{l}\text { Wuhan and } \\
\text { Beijing, China }\end{array}$ & $\begin{array}{c}\text { near manufacture } \\
\text { site/urban }\end{array}$ & 2015 & 9 & n.a. & n.a. & n.a. & n.a. & $2.8-13$ & $0.4-0.6$ & 9 \\
\hline $\begin{array}{l}\text { Stockholm and } \\
\text { Råö, Sweden }\end{array}$ & urban & $\begin{array}{l}2015- \\
2016\end{array}$ & 21 & n.a. & n.a. & n.a. & n.a. & $0.3-1.4$ & $0.1-2.0$ & 9 \\
\hline $\begin{array}{l}\text { Eastern and } \\
\text { central China }\end{array}$ & $\begin{array}{l}\text { remote/urban/near } \\
\text { textile manufacturer }\end{array}$ & 2010 & 19 & n.a. & n.a. & n.a. & n.a. & $0.7-88$ & $\begin{array}{c}<\mathrm{MQL}- \\
16\end{array}$ & 10 \\
\hline $\begin{array}{c}\text { across mainland } \\
\text { China }\end{array}$ & & 2019 & 39 & n.a. & n.a. & n.a. & n.a. & $0.30-100$ & $0.90-13$ & 11 \\
\hline Dalian, China & urban/coastal & $\begin{array}{l}2006- \\
2007\end{array}$ & 2 & n.a. & n.a. & n.a. & n.a. & $33-41$ & 9.9-110 & 12 \\
\hline \multicolumn{11}{|l|}{ Dry Deposition } \\
\hline Wilmington, NC & urban/coastal & $\begin{array}{c}2018- \\
2020\end{array}$ & 25 & $\begin{array}{c}<\mathrm{MQL}- \\
22\end{array}$ & $\begin{array}{c}<\mathrm{MQL}- \\
0.9\end{array}$ & $\begin{array}{c}<\mathrm{MQL}- \\
2.1\end{array}$ & $\begin{array}{c}<\mathrm{MQL}- \\
1.6\end{array}$ & $\begin{array}{c}<\mathrm{MQL}- \\
0.5\end{array}$ & $\begin{array}{c}<\mathrm{MQL}- \\
16\end{array}$ & This study \\
\hline $\begin{array}{l}\text { Dordrecht, } \\
\text { Netherland }\end{array}$ & $\begin{array}{c}\text { within } 3 \mathrm{~km} \text { from a } \\
\text { fluorochemical } \\
\text { manufacturer }\end{array}$ & & 12 & n.a. & n.a. & n.a. & $\begin{array}{c}<0.1-86 \\
\mathrm{ng} / \mathrm{g} \\
\mathrm{ww}^{*}\end{array}$ & $\begin{array}{c}<0.7-28 \\
\mathrm{ng} / \mathrm{g} \\
\mathrm{ww}^{*}\end{array}$ & $\begin{array}{c}<0.4 \mathrm{ng} / \mathrm{g} \\
\mathrm{ww}^{*}\end{array}$ & 13 \\
\hline
\end{tabular}




\begin{tabular}{|c|c|c|c|c|c|c|c|c|c|c|}
\hline Tianjin, China & $\begin{array}{l}\text { urban, suburban, } \\
\text { rural, remote }\end{array}$ & 2013 & & n.a. & n.a. & n.a. & n.a. & n.a. & $\begin{array}{c}1.4-5.7 \\
\mathrm{ng} / \mathrm{g}\end{array}$ & 14 \\
\hline $\begin{array}{l}\text { Bohai and } \\
\text { Yellow Sea, } \\
\text { China }\end{array}$ & $\begin{array}{l}\text { near fluorochemical } \\
\text { manufacturers }\end{array}$ & 2016 & 16 & n.a. & n.a. & n.a. & n.a. & $0.6-7.4^{* *}$ & $\begin{array}{l}0.19- \\
1.1^{* *}\end{array}$ & 15 \\
\hline \multicolumn{11}{|c|}{ Total Deposition as Snow or ice Core } \\
\hline $\begin{array}{c}\text { Livingston } \\
\text { Island, Antarctica }\end{array}$ & remote/coastal & $\begin{array}{l}2014- \\
2015\end{array}$ & 19 & n.a. & n.a. & n.a. & n.a. & $0.3-1.3$ & $\begin{array}{c}0.02- \\
0.1\end{array}$ & 16 \\
\hline $\begin{array}{l}\text { Devon Island, } \\
\text { Nunavut, Canada }\end{array}$ & remote & 2015 & & & & & & 0.11 & 0.052 & 17 \\
\hline Dalian, China & urban/coastal & $\begin{array}{l}2006- \\
2007\end{array}$ & 21 & n.a. & n.a. & n.a. & n.a. & $8.1-66$ & $27-550$ & 12 \\
\hline
\end{tabular}

*Dry deposition on grasses and leaves, units are ng/g wet weight

** These are estimation based on particle-phase concentrations in the air 
Table. S8. Annual Flux (ng $\mathrm{m}^{-2}$ ) of PFASs by dry and wet deposition, and flux ratios of wet deposition to dry deposition.

\begin{tabular}{llrr}
\hline & Flux by Dry & \multicolumn{2}{l}{ Flux by Wet } \\
Compound & Deposition $\left(\mathrm{ng} \mathrm{m}^{-2}\right)$ & Deposition $\left(\mathrm{ng} \mathrm{m}^{-2}\right)$ & Flux Ratio \\
\hline PFMOAA & 200 & 2400 & 12 \\
PFMOPrA & 63 & 2300 & 36 \\
PFMOBA & 70 & 2000 & 29 \\
HFPO.DA & 150 & 5700 & 39 \\
PFOA & 55 & 1300 & 24 \\
PFOS & 350 & 18000 & 50 \\
\hline Total & 880 & 31000 & 36 \\
\hline
\end{tabular}


<smiles>O=C(O)C(F)(F)OC(F)(F)F</smiles>

PFMOAA

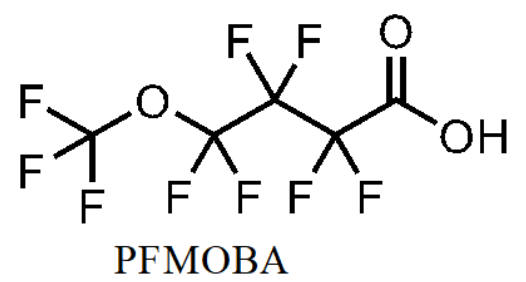

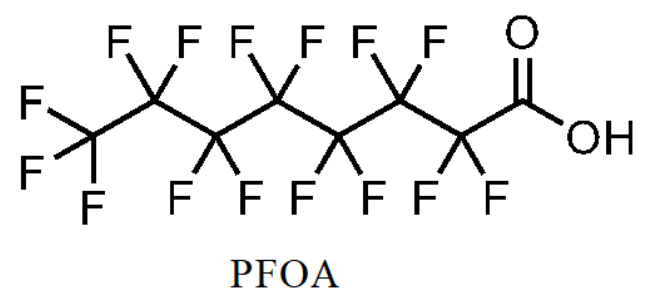

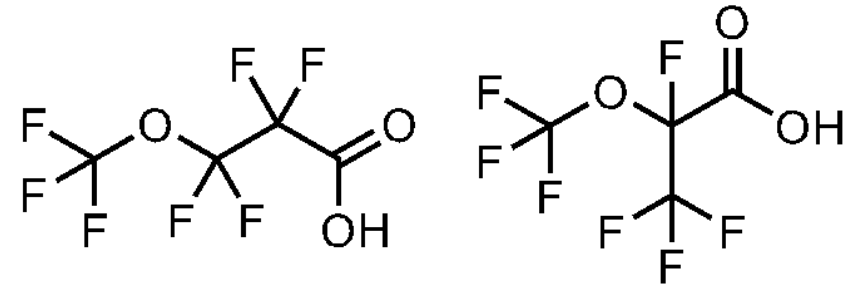

PFMOPrA / PMPA
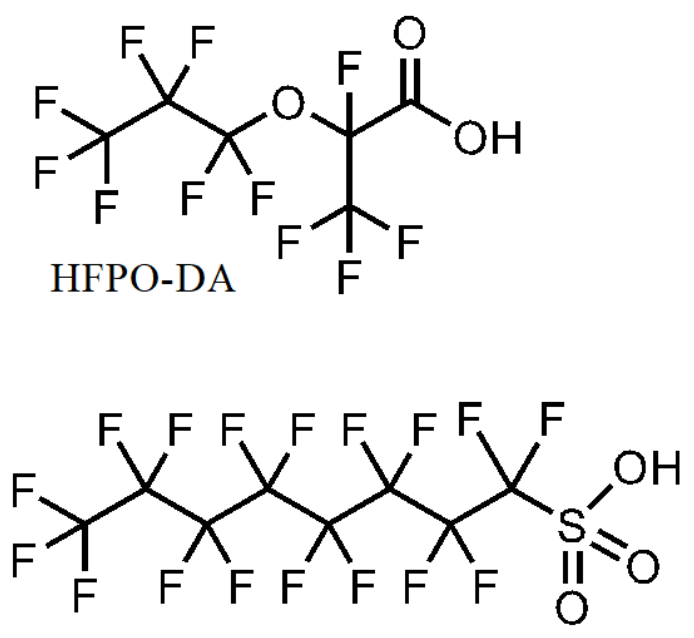

PFOS

Figure S1. Molecular structures of PFASs quantified in this study. 

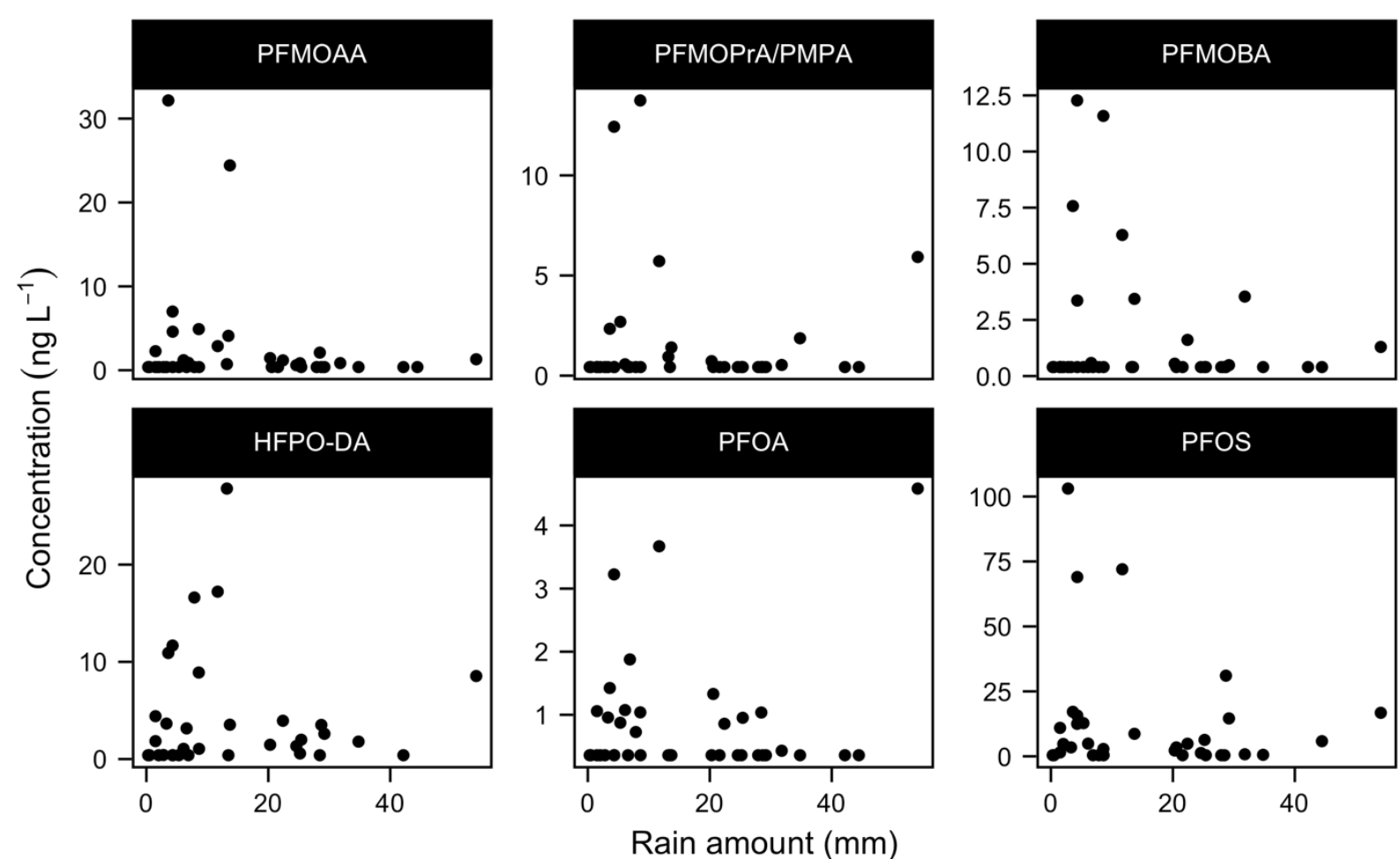

Figure S2. PFAS concentrations in wet deposition as a function of rain amount. 

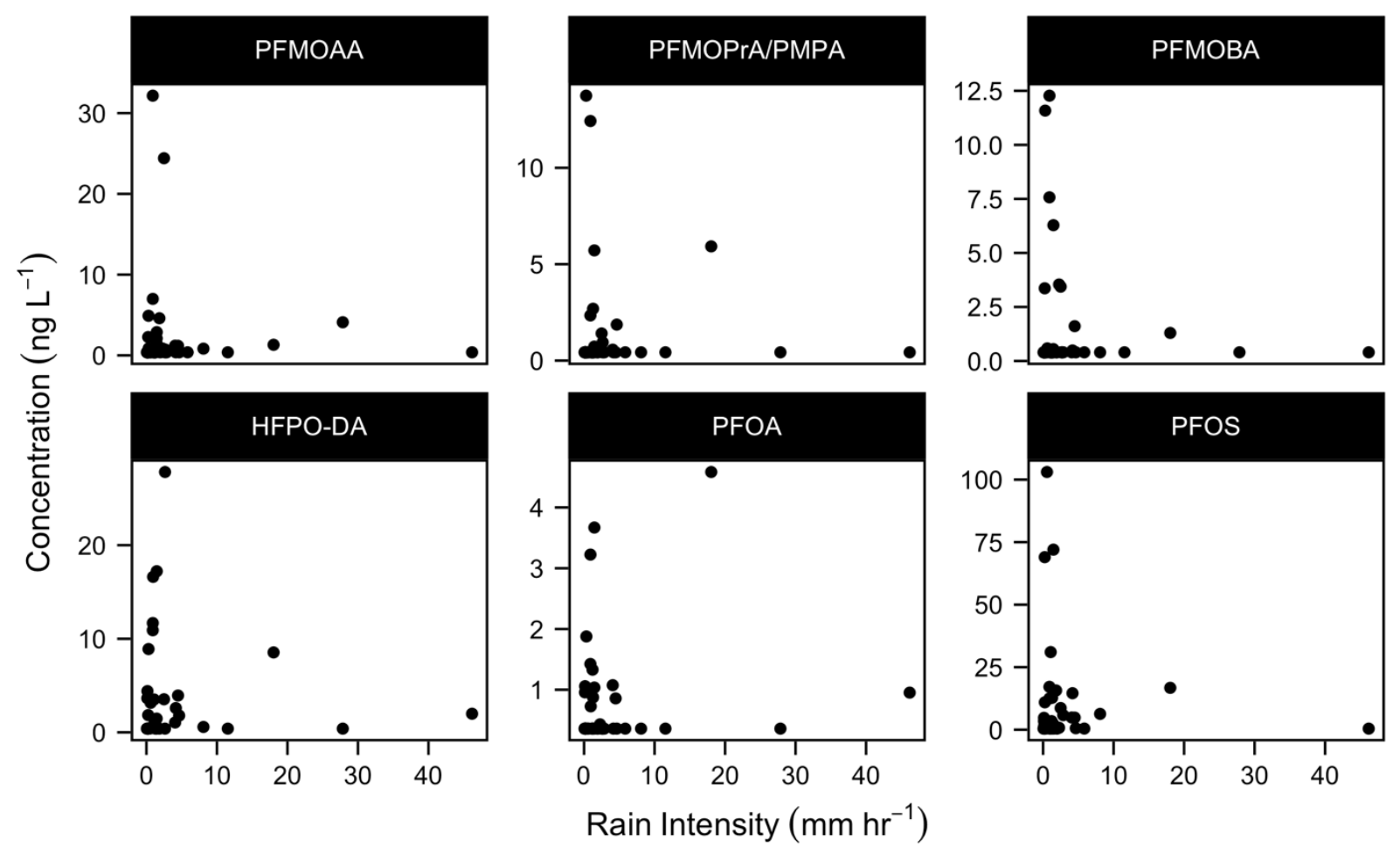

Figure S3. PFAS concentrations in wet deposition as a function of rain intensity. 

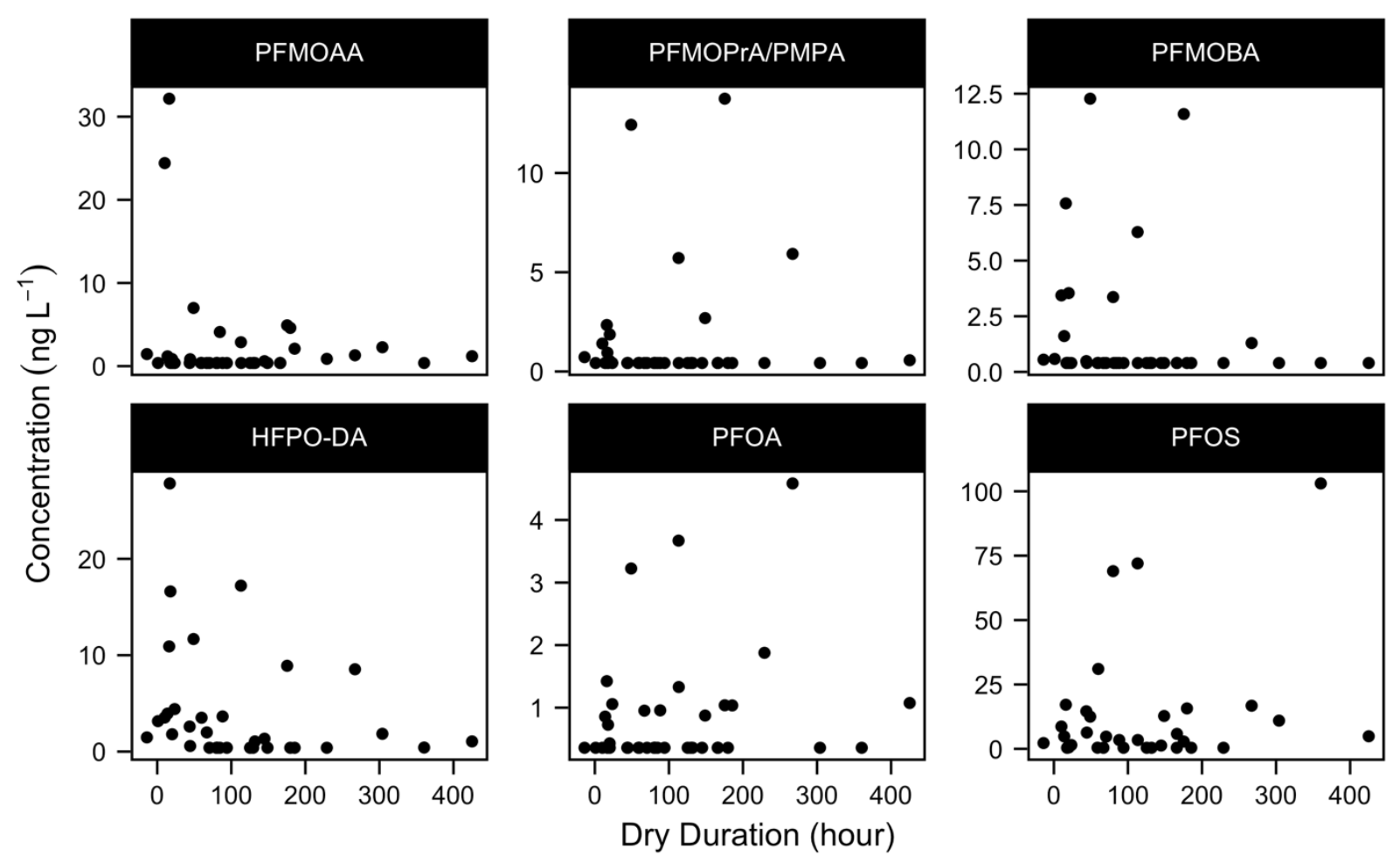

Figure S4. PFAS concentrations in wet deposition as a function of dry duration before starting rain. 


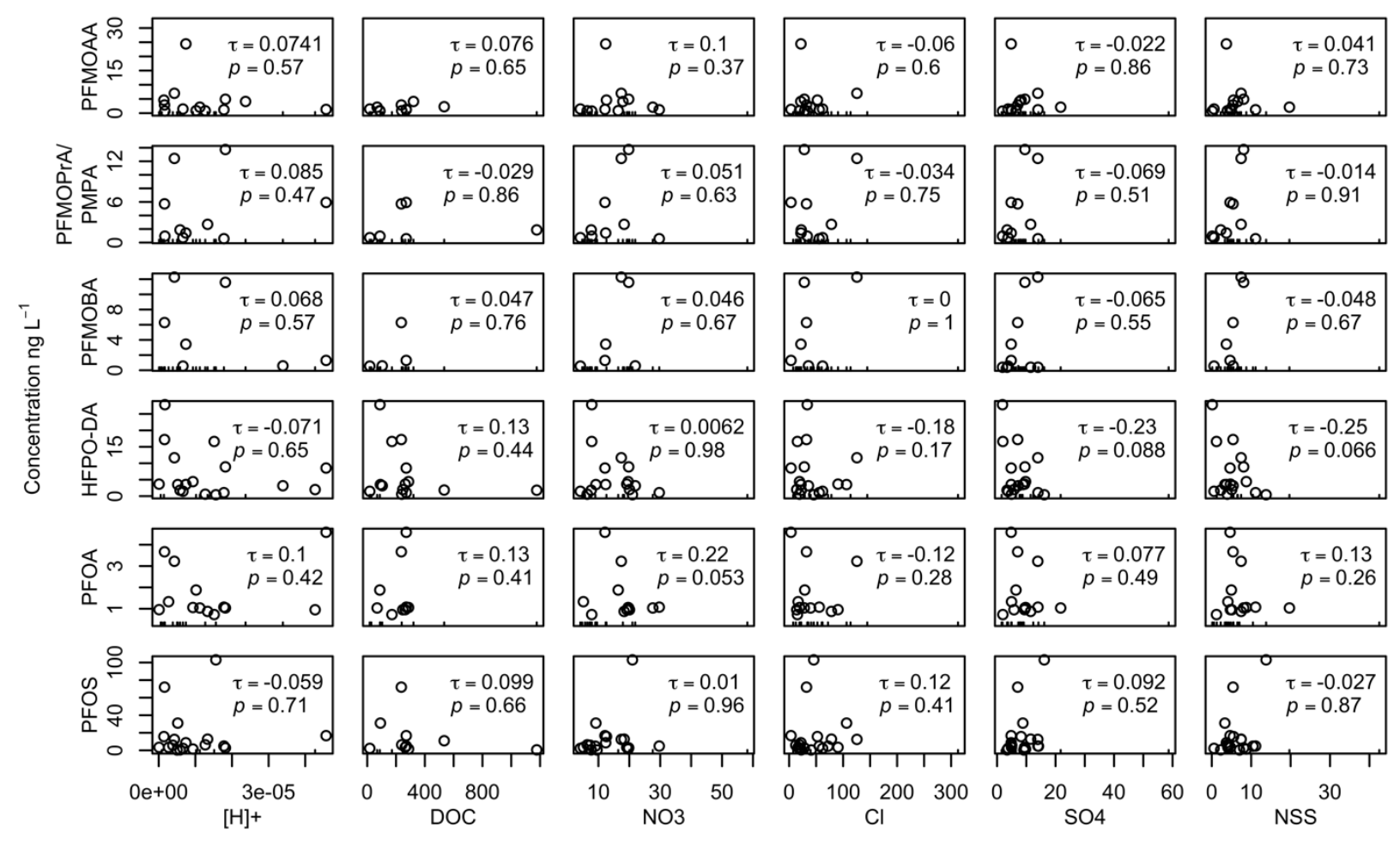

Figure S5. Correlation of PFAS concentrations in wet deposition with concentrations of hydrogen ion in $\mathrm{M}$, dissolved organic carbon (DOC) in $\mu \mathrm{M}$, anions $\left(\mathrm{Cl}^{-}, \mathrm{NO}_{3}^{-}\right.$, and $\left.\mathrm{SO}_{4}{ }^{2-}\right)$ in $\mu \mathrm{M}$, and non-seasalt sulfate (NSS) in $\mu \mathrm{M}$. No significant correlation was found (Kendall's $\tau$; p-value $>0.053)$. 

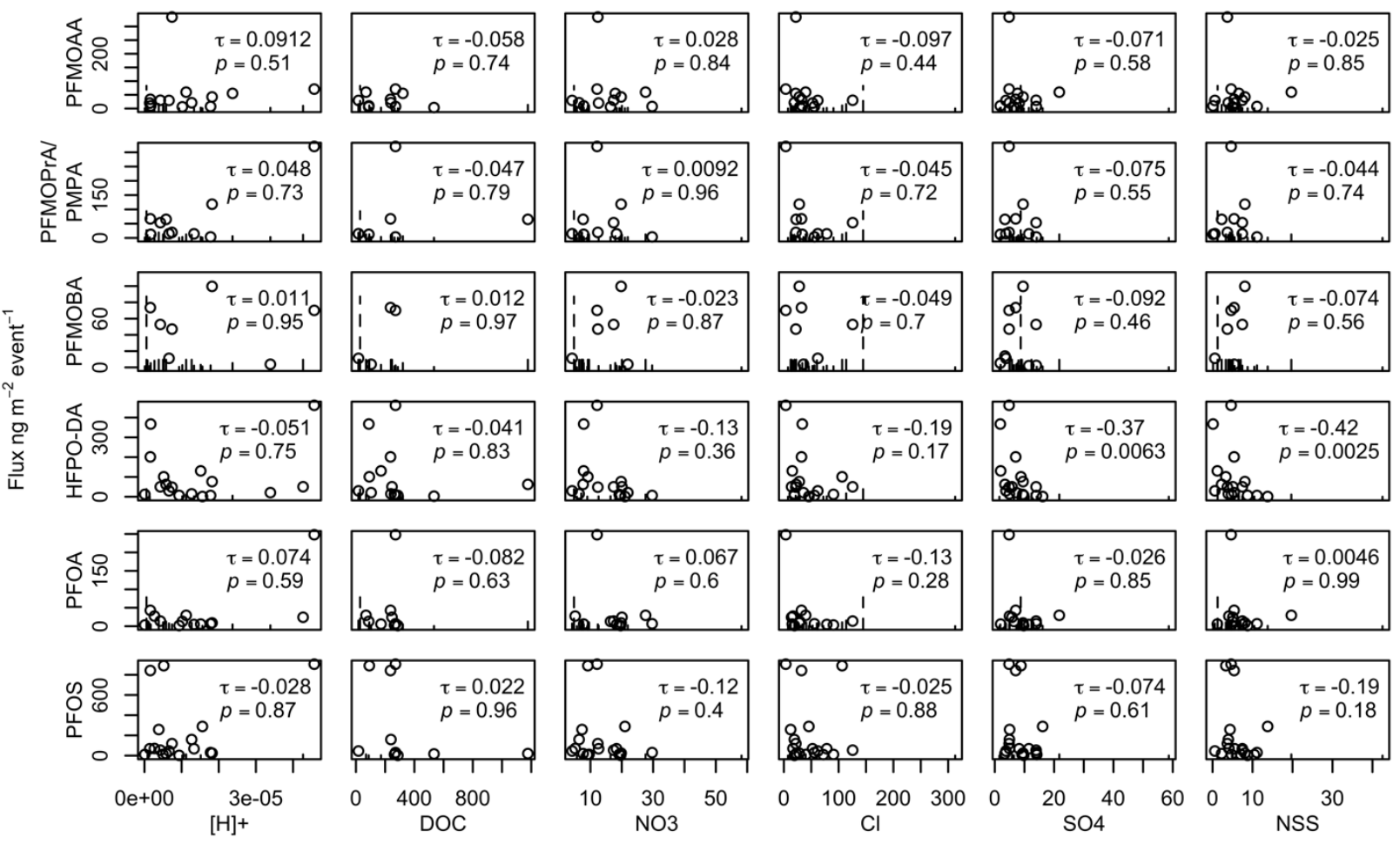

Figure S6. Correlation of PFAS flux by wet deposition with concentrations of hydrogen ion in $\mathrm{M}$, dissolved organic carbon (DOC), anions (Cl- , NO3- , and SO42- ), and non-seasalt sulfate (NSS) in $\mu \mathrm{M}$. A negative correlation was found between HFPO-DA and NSS and sulfate. No significant correlation was found for others (Kendall's $\tau$; p-value $>0.17$ ).

References

(1) Sun, M.; Arevalo, E.; Strynar, M.; Lindstrom, A.; Richardson, M.; Kearns, B.; Pickett, A.; Smith, C.; Knappe, D. R. U. Legacy and Emerging Perfluoroalkyl Substances Are Important Drinking Water Contaminants in the Cape Fear River Watershed of North Carolina.

Environ. Sci. Technol. Lett. 2016, 3 (12), 415-419.

https://doi.org/10.1021/acs.estlett.6b00398.

(2) Pan, Y.; Zhang, H.; Cui, Q.; Sheng, N.; Yeung, L. W. Y.; Sun, Y.; Guo, Y.; Dai, J. Worldwide Distribution of Novel Perfluoroether Carboxylic and Sulfonic Acids in Surface Water. Environ. Sci. Technol. 2018, 52 (14), 7621-7629. https://doi.org/10.1021/acs.est.8b00829.

(3) Stein, A. F.; Draxler, R. R.; Rolph, G. D.; Stunder, B. J. B.; Cohen, M. D.; Ngan, F. NOAA's HYSPLIT Atmospheric Transport and Dispersion Modeling System. Bull. Amer. Meteor. Soc. 2015, 96 (12), 2059-2077. https://doi.org/10.1175/BAMS-D-14-00110.1. 
(4) Shimizu, M. S.; Summerlin, S. L.; Felix, J. D.; Halls, J.; Avery, G. B. Jr.; Kieber, R. J.; Lane, C. S.; Mead, R. N.; Willey, J. D. Variable Ethanol Concentrations and Stable Carbon Isotopes Reveal Anthropogenic Ethanol Contributions to Rainwater. Atmospheric Environment 2020, 234, 117578. https://doi.org/10.1016/j.atmosenv.2020.117578.

(5) Willey, J. D.; Kieber, R. J.; Eyman, M. S.; Avery, G. B., Jr. Rainwater Dissolved Organic Carbon: Concentrations and Global Flux. Global Biogeochemical Cycles 2000, 14 (1), 139148. https://doi.org/10.1029/1999GB900036.

(6) Helsel, D. R. Fabricating Data: How Substituting Values for Nondetects Can Ruin Results, and What Can Be Done about It. Chemosphere 2006, 65 (11), 2434-2439. https://doi.org/10.1016/j.chemosphere.2006.04.051.

(7) Pike, K. A.; Edmiston, P. L.; Morrison, J. J.; Faust, J. A. Correlation Analysis of Perfluoroalkyl Substances in Regional U.S. Precipitation Events. Water Research 2021, 190, 116685. https://doi.org/10.1016/j.watres.2020.116685.

(8) Gewurtz, S. B.; Bradley, L. E.; Backus, S.; Dove, A.; McGoldrick, D.; Hung, H.; DryfhoutClark, H. Perfluoroalkyl Acids in Great Lakes Precipitation and Surface Water (2006-2018) Indicate Response to Phase-Outs, Regulatory Action, and Variability in Fate and Transport Processes. Environ. Sci. Technol. 2019, 53 (15), 8543-8552. https://doi.org/10.1021/acs.est.9b01337.

(9) Johansson, J. H.; Shi, Y.; Salter, M.; Cousins, I. T. Spatial Variation in the Atmospheric Deposition of Perfluoroalkyl Acids: Source Elucidation through Analysis of Isomer Patterns. Environ. Sci.: Processes Impacts 2018, 20 (7), 997-1006. https://doi.org/10.1039/C8EM00102B.

(10) Zhao, L.; Zhou, M.; Zhang, T.; Sun, H. Polyfluorinated and Perfluorinated Chemicals in Precipitation and Runoff from Cities Across Eastern and Central China. Archives of Environmental Contamination and Toxicology; New York 2013, 64 (2), 198-207. http://dx.doi.org.liblink.uncw.edu/10.1007/s00244-012-9832-x.

(11) Chen, H.; Zhang, L.; Li, M.; Yao, Y.; Zhao, Z.; Munoz, G.; Sun, H. Per- and Polyfluoroalkyl Substances (PFASs) in Precipitation from Mainland China: Contributions of Unknown Precursors and Short-Chain (C2C3) Perfluoroalkyl Carboxylic Acids. Water Research 2019, 153, 169-177. https://doi.org/10.1016/j.watres.2019.01.019.

(12) Liu, W.; Jin, Y.; Quan, X.; Sasaki, K.; Saito, N.; Nakayama, S. F.; Sato, I.; Tsuda, S. Perfluorosulfonates and Perfluorocarboxylates in Snow and Rain in Dalian, China. Environment International 2009, 35 (4), 737-742. https://doi.org/10.1016/j.envint.2009.01.016.

(13) Brandsma, S. H.; Koekkoek, J. C.; van Velzen, M. J. M.; de Boer, J. The PFOA Substitute GenX Detected in the Environment near a Fluoropolymer Manufacturing Plant in the Netherlands. Chemosphere 2019, 220, 493-500. https://doi.org/10.1016/j.chemosphere.2018.12.135.

(14) Yao, Y.; Sun, H.; Gan, Z.; Hu, H.; Zhao, Y.; Chang, S.; Zhou, Q. Nationwide Distribution of Per- and Polyfluoroalkyl Substances in Outdoor Dust in Mainland China From Eastern to Western Areas. Environ. Sci. Technol. 2016, 50 (7), 3676-3685. https://doi.org/10.1021/acs.est.6b00649.

(15) Fang, X.; Wang, Q.; Zhao, Z.; Tang, J.; Tian, C.; Yao, Y.; Yu, J.; Sun, H. Distribution and Dry Deposition of Alternative and Legacy Perfluoroalkyl and Polyfluoroalkyl Substances in the 
Air above the Bohai and Yellow Seas, China. Atmospheric Environment 2018, 192, 128135. https://doi.org/10.1016/j.atmosenv.2018.08.052.

(16) Casal, P.; Zhang, Y.; Martin, J. W.; Pizarro, M.; Jiménez, B.; Dachs, J. Role of Snow Deposition of Perfluoroalkylated Substances at Coastal Livingston Island (Maritime Antarctica). Environ. Sci. Technol. 2017, 51 (15), 8460-8470. https://doi.org/10.1021/acs.est.7b02521.

(17) Pickard, H. M.; Criscitiello, A. S.; Spencer, C.; Sharp, M. J.; Muir, D. C. G.; De Silva, A. O.; Young, C. J. Continuous Non-Marine Inputs of per- and Polyfluoroalkyl Substances to the High Arctic: A Multi-Decadal Temporal Record. Atmos. Chem. Phys. 2018, 18 (7), 50455058. https://doi.org/10.5194/acp-18-5045-2018. 